\title{
Acoustically Driven Cortical $\delta$ Oscillations Underpin Prosodic Chunking
}

\author{
(D). M. Rimmele, ${ }^{1,2}$-D. Poeppel, ${ }^{1,2,3}$ and O. Ghitza ${ }^{1,4}$
}

https://doi.org/10.1523/ENEURO.0562-20.2021

${ }^{1}$ Department of Neuroscience, Max Planck Institute for Empirical Aesthetics, Frankfurt am Main 60322, Germany,

${ }^{2}$ Max Planck NYU Center for Language, Music, and Emotion, Frankfurt am Main, Germany, New York, NY,

${ }^{3}$ Department of Psychology and Center for Neural Science, New York University, New York, NY 10003, and

${ }^{4}$ Department of Biomedical Engineering and Hearing Research Center, Boston University, Boston, MA 02215

\begin{abstract}
Oscillation-based models of speech perception postulate a cortical computational principle by which decoding is performed within a window structure derived by a segmentation process. Segmentation of syllable-size chunks is realized by a $\theta$ oscillator. We provide evidence for an analogous role of a $\delta$ oscillator in the segmentation of phrase-sized chunks. We recorded magnetoencephalography (MEG) in humans, while participants performed a target identification task. Random-digit strings, with phrase-long chunks of two digits, were presented at chunk rates of 1.8 or $2.6 \mathrm{~Hz}$, inside or outside the $\delta$ frequency band (defined here to be $0.5-2 \mathrm{~Hz}$ ). Strong periodicities were elicited by chunk rates inside of $\delta$ in superior, middle temporal areas and speechmotor integration areas. Periodicities were diminished or absent for chunk rates outside $\delta$, in line with behavioral performance. Our findings show that prosodic chunking of phrase-sized acoustic segments is correlated with acoustic-driven $\delta$ oscillations, expressing anatomically specific patterns of neuronal periodicities.
\end{abstract}

Key words: auditory; $\delta$-band; oscillations; prosodic chunking

\section{Significance Statement}

Oscillation-based models of speech perception postulate a cortical computational principle by which decoding is performed within a time-varying window structure, synchronized with the input on multiple time scales. At prelexical level, cycles of a flexible $\theta$ oscillator, locked to the input syllabic rhythm, constitute the syllabic windows. We find that the presence of cortical $\delta$ oscillations correlates with whether or not an input phrase-sized chunk rate is inside the $\delta$ range. This suggests that at the phrase time scale, a $\delta$ oscillator could play a role analogous to that of the $\theta$ oscillator at the syllable level. The segmentation process is realized by a flexible $\delta$ oscillator locked to the input rhythm, with $\delta$ cycles constituting phrase-sized windows.

\section{Introduction}

Naturally spoken language is a stream of connected sounds, and although the speech acoustics contain no cues regarding the beginning or end of linguistic units a combination of interleaved cues (e.g., pauses, intonation, syllabic stress) are embedded in the acoustic stream. Information, broadly termed "accentuation" (e.g., intonation, stress, pauses), is used by listeners to indicate boundaries associated with linguistic units (Aubanel et al., 2016; Oganian and Chang, 2019). The process by which

Received December 27, 2020; accepted May 9, 2021; First published June 3, 2021.

The authors declare no competing financial interests. the input signal is partitioned into temporal segments to be linked to a variety of linguistic levels of abstraction (ranging from phonetic segments to syllables to words and, ultimately, prosodic and intonational phrases) is called "segmentation."

The segmentation process has been shown to operate on time intervals associated with syllables (up to $\sim 250 \mathrm{~ms}$; Brungart et al., 2007; Ghitza and Greenberg, 2009; Doelling et al., 2014; Kösem et al., 2018), and a similar process has been suggested to operate on the phrasal

Author contributions: J.M.R., D.P., and O.G. designed research; J.M.R. performed research; O.G. contributed unpublished reagents/analytic tools; J.M.R. and O.G. analyzed data; J.M.R., D.P., and O.G. wrote the paper. 
level (0.5-2 s; Ding et al., 2016; Ghitza, 2017; Martin and Doumas, 2017; Keitel et al., 2018). At the syllabic level, perceptual segmentation, or chunking, is by and large a prelexical process. Oscillation-based models propose that this segmentation is realized by flexible $\theta$ oscillations aligning their phase to the input syllabic rhythm ("speech tracking"), where the $\theta$ cycles mark the speech chunks to be decoded (Poeppel, 2003; Lakatos et al., 2005, 2019; Ahissar and Ahissar, 2005; Ding and Simon, 2009; Ghitza, 2011; Giraud and Poeppel, 2012; Peelle and Davis, 2012; Gross et al., 2013; Haegens and Zion Golumbic, 2018; Rimmele et al., 2018a; Assaneo et al., 2020; Hovsepyan et al., 2020; Pittman-Polletta et al., 2021). At the phrase level, phrase rhythm can affect segmentation (Gee and Grosjean, 1983; Martin, 2015; Deniz and Fodor, 2019; Hilton and Goldwater, 2020). There have been various studies aiming to quantify phrase length and rhythmicity (Clifton et al., 2006; Breen, 2018; Deniz and Fodor, 2019), suggesting that typical intonational phrases are about $1 \mathrm{~s}$ in duration (Auer et al., 1999; Inbar et al., 2020; Stehwien and Meyer, 2021). More specifically, the duration of intonational phrases spans a range between $\sim 0.5$ and $1 \mathrm{~s}$ in English (slightly faster in some other languages; Inbar et al., 2020; Stehwien and Meyer, 2021). Prosodic segmentation (here also termed "prosodic chunking") is based on intonation units that contain specific prosodic cues (such as pauses or pitch contour), which can pace the information flow at the phrasal time scale (Shattuck-Hufnagel and Turk, 1996; Inbar et al., 2020). The extent to which phrase level rhythmic structure supports segmentation and structural parsing was not widely studied. Here, we investigate the neural processing of rhythmic structure at the phrasal scale by analyzing how individuals' group single digits into "phrase-sized" digit chunks. What kind of neuronal mechanism can realize this chunking process?

Cortical $\delta$ oscillators, with a frequency range $(\sim 0.5-$ $2 \mathrm{~Hz}$ ) that corresponds to the phrasal time scale, were shown to be elicited during phrasal processing of speech or chunking processes at the phrasal scale (Buiatti et al., 2009; Ding et al., 2016; Bonhage et al., 2017; Meyer et al., 2017; Keitel et al., 2018; Boucher et al., 2019). $\delta$ Was observed in the posterior superior temporal, the inferior frontal, precentral, and temporal-parietal cortex using ECoG (Ding et al., 2016), and using EEG at bilateral middle and superior temporal areas (also fusiform gyrus; Bonhage et al., 2017) and at frontotemporal sites (Boucher et al., 2019). [Recall the ambiguous definition of the $\delta$ range in the literature, which covers a range of overlapping

This work was supported by the Max Planck Institute for Empirical Aesthetics, Max Planck NYU Center for Language, Music, and Emotion (CLaME), and the United States Air Force Office of Scientific Research Grant FA9550-18-1-0055.

Acknowledgements: We thank Simone Franz and Daniela van Hinsberg for help with the data recording.

Correspondence should be addressed to J. M. Rimmele at johanna. rimmele@ae.mpg.de.

https://doi.org/10.1523/ENEURO.0562-20.2021

Copyright (C) 2021 Rimmele et al.

This is an open-access article distributed under the terms of the Creative Commons Attribution 4.0 International license, which permits unrestricted use, distribution and reproduction in any medium provided that the original work is properly attributed.

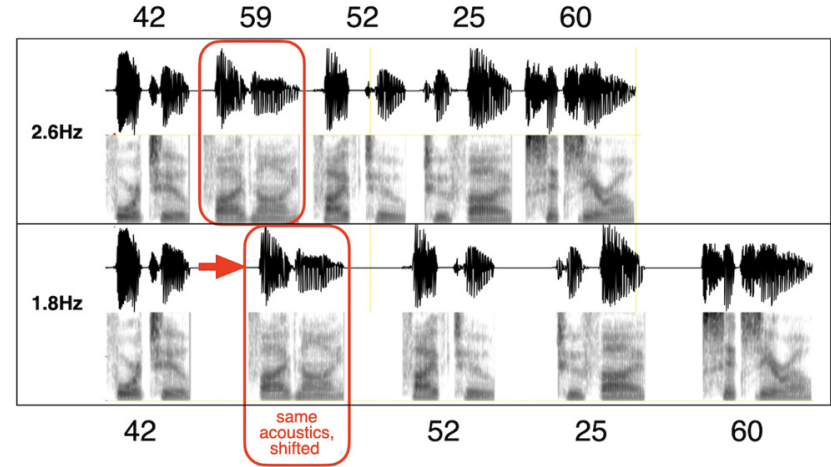

Figure 1. Chunk patterns and chunk rates for the 10-digit digit string 4259522560 . The chunk pattern is 2222 , with chunk rates of $1.8 \mathrm{~Hz}$ (inside $\delta$ ) and $2.6 \mathrm{~Hz}$ (outside). Each chunk was synthesized as a two-digit unit, using the AT\&T Text-to-Speech System accentuation (see text). Note that a particular two-digit chunk has the same acoustics, regardless of whether it occurs in the 1.8- or 2.6- $\mathrm{Hz} 2222$ chunk condition (red box). The 1.8$\mathrm{Hz}$ stimulus is generated by increasing the gap between the chunks (with identical chunk acoustics).

frequency bands inside the $0.5-$ to $4-\mathrm{Hz}$ frequency range (Bonhage et al., 2017; Keitel et al., 2018; Bröhl and Kayser, 2020). Since we are interested in the segmentation of phrasal chunks, which in English are $\sim 0.5-1 \mathrm{~s}$ long (Miller, 1962; Inbar et al., 2020), we opted to define the $\delta$ frequency band to be $0.5-2 \mathrm{~Hz}$.]

And behaviorally, it has been shown that performance is impaired when the chunk rate is outside of the $\delta$ range (Ghitza, 2017). These findings suggest a role of neuronal oscillatory mechanisms in the $\delta$ range in chunking at a phrasal time scale (see also Martin and Doumas, 2017; Ghitza, 2020). Little is known, however, about the brain areas that may recruit chunking-related $\delta$ oscillations.

Here, we focus on the cortical mechanism that may be involved in acoustic-driven segmentation at a phrasal time scale, using sequences of digit chunks (with a minimal amount of content). We test the hypothesis that the decoding process is guided by a $\delta$ oscillator locked to the accentuation acoustic cues (Ghitza, 2017) by recording magnetoencephalography (MEG) data while participants performed a digit retrieval task. The digits in the string were grouped into chunks, with chunk rates either inside or outside to the $\delta$ frequency range (Fig. 1). The experiment addresses two questions: (1) Do elicited $\delta$ brain waves correlate with behavior, such that impaired performance in digit retrieval occurs if the chunk rate is outside of the $\delta$ range? (2) Where in the auditory pathway do those neuronal oscillations occur?

Our data show that in superior and middle temporal areas and in speech-motor planning and integration areas (IFG, PC, SMG), robust neural $\delta$ periodicities were elicited by chunk rates inside of the $\delta$ range but were diminished when the chunk rate was outside of the $\delta$ range, in line with behavioral performance. In speech-motor integration areas (SMG) and areas implicated in processing word form and meaning (middle temporal gyrus; MTG), periodicity was present albeit diminished even for chunk rates inside the $\delta$ range. The $\delta$ periodicities were acoustically 
driven, in sync with the input as long as the acoustic chunk rate was inside $\delta . \delta$ Periodicities were diminished for chunk rates outside $\delta$, although sufficient acoustic cues for chunking were present in all conditions. Thus, the failure to track the input-chunk-rate when it was outside of the $\delta$ range was not caused by insufficient acoustic cues but seems due to neuronal circuitry characteristics constraining the tracking of the chunks.

\section{Materials and Methods}

\section{Participants}

The data from 19 healthy right-handed (Oldfield, 1971) mean score: 75.22 , SD: 18.08) participants were included in the study (mean age: 24.89 years, SD: $3.54 ; f=14$ ). Human subjects were recruited from the local community in Frankfurt. Two participants were excluded because of technical issues, and one participant because of outlier performance (i.e., performance $<$ mean performance, 2 $\mathrm{SD})$. Individual MRI scans were recorded for all except for two participants who did not fulfill the MRI scanning criteria. All participants gave written informed consent for participating in the study and received monetary compensation. The study was approved by the local ethics committee of the University Hospital Frankfurt (SG2-02-0128).

\section{Digit string stimuli}

We used 10-digit long stimuli where we manipulated the pauses in-between digits according to the experimental conditions. We opted for digit sequences -material that is semantically unpredictable at the digit-chunk level (i.e., while semantic information is present at the single digit level, no semantic/contextual information is present at the digit-chunk level), to minimize the bottom-up/ top-down interaction that is in play in setting perceptual boundaries for digit-chunks. The digit sequences were grouped into chunks, with a chunk pattern termed 2222. For example, the 2222 pattern of the sequence 4259522560 is [42 595225 60]. Digits were presented as single digits, i.e., 42 was read as four-two and not as forty-two.

We used two chunk rates: $1.8 \mathrm{~Hz}$ (inside the $\delta$ range) and $2.6 \mathrm{~Hz}$ (at the outside border of the $\delta$ range, referred to as "outside"), termed conditions "1.8 Hz" and "2.6 Hz" (Fig. 1). Note that a third condition was used, which is not reported here. The condition was a "no-chunk" condition where digit chunks were presented at the rate of $2.6 \mathrm{~Hz}$. However, besides top-down chunking information (provided by the instructions), there were no acoustic chunking cues. The neuronal findings resemble that of the 2.6$\mathrm{Hz}$ chunking condition, confirming the main claims of this paper. They are reported elsewhere (Rimmele et al., 2020).

\section{Corpus}

The text material comprised 100 10-digit long text strings. Stimuli were generated by using the AT\&T Textto-Speech System with the American English female speaker Crystal. [The AT\&T-TTS system (http://www. wizzardsoftware.com/text-to-voice.php) uses a form of concatenative synthesis based on a unit-selection process, where the units are cut from a large, high-quality, prerecorded natural voice fragments. The system produces natural-sounding, highly intelligible spoken material with a realistic prosodic rhythm, with accentuation defined by the system-internal prosodic rules, and is considered to have some of the finest quality synthesis of any commercial product.] To generate stimuli with a 2222 chunk pattern, we first created a two-digit chunk vocabulary as follows. For each doublet of digits that exists in the 100 text strings, a naturally sounding two-digit chunk waveform was generated (naturalness was obtained by the AT\&T system accentuation rules) resulting in a chunkvocabulary. For a given text string, a 2222 10-digit stimulus was generated by concatenating the corresponding five two-digit chunk waveforms pulled from the chunk-vocabulary. The chunk rate was set by adjusting the gap duration in between two successive chunks, resulting in a stimulus with a temporal structure but without any contextual cues at the digit-chunk level. To enable the generation of stimuli with chunk rates greater than the $\delta$ frequency upper bound (at $2.6 \mathrm{~Hz}$ ), the waveforms in all conditions were time compressed by a factor of 2.5, just below the auditory channel capacity (Ghitza, 2014). The duration of the 10-digit stimuli varied across conditions; for the 1.8- $\mathrm{Hz}$ condition: mean $=2.61 \mathrm{~s}(\mathrm{VAR}=85.6 \mathrm{~ms})$, and for the $2.6-\mathrm{Hz}$ condition: mean $=1.99 \mathrm{~s}(\mathrm{VAR}=85.6$ $\mathrm{ms})$.

For each of the 20010 -digit stimuli (100 stimuli for each of the 1.8- and $2.6-\mathrm{Hz}$ conditions) a trial was created by concatenating the following waveform sequence: [one digit trial-count] [20-ms-long gap] [10-digit string] [500ms-long gap] [two-digit target], resulting in one concatenated waveform per trial with durations that varied across trials and conditions. The 200 trials were scrambled, and the resulting pool of trials was divided into blocks, 50 trials per block. A jittered intertrial interval of 3-4.5 s was presented between trials. Overall, two different sets of stimuli were used.

\section{Task}

Behavioral and MEG data were collected while participants performed a digit retrieval task, in the form of an adapted Sternberg target identification task (Sternberg, 1966; target ID task from here on): listeners heard a 10digit stimulus followed by a two-digit-long target, and were asked to indicate whether or not the target was part of the utterance. A target position was always within a chunk. Note that the task is suitable for probing the role of acoustic segmentation in a memory retrieval task: a successful yes/no decision depends on how faithful the recognized chunk objects are, generated by a decoding process that, by hypothesis, depends on the goodness of segmentation.

\section{Procedure and paradigm}

Participants were seated in front of a board for instructions in the MEG testing booth. Binaurally insert earplugs 
(E-A-RTONE Gold 3A Insert Earphones, Ulrich Keller Medizin-Technik) were used for stimulus presentation. Two button boxes (Current Designs) were used to record participants' responses. The Psychophysics Toolbox (Brainard, 1997) was used to run the experiment. During the experiment, on each trial participants fixated the screen center (fixation cross) while listening to the digit sequences. The sounds were presented at a comfortable loudness level ( $\sim 70 \mathrm{~dB}$ SPL), which remained unchanged throughout the experiment. Overall, the experiment lasted $\sim 2.5 \mathrm{~h}$, including preparation time, recording time, breaks, and postrecording questionnaires. Participants were presented with the task requirements. They were instructed that all sequences comprise concatenated chunks of twodigits. Before the experiment, all participants performed a short training of three trials (with feedback) to familiarize themselves with the stimuli and task. Participants were asked to indicate by button press (yes/no response; with the response hand balanced across participants; yes-hand right: $N=12$ ) whether or not the target was part of the preceded utterance.

\section{MRI and MEG data acquisition}

A 3 Tesla scanner (Siemens Magnetom Trio, Siemens) was used to record individual T1-weighted MRIs. MEG recordings were performed on a 269-channel wholehead MEG system (Omega 2000, CTF Systems Inc.) in a magnetically shielded booth. Data were acquired with a sampling rate of $1200 \mathrm{~Hz}$, online denoising (higher-order gradiometer balancing) and online low pass filtering (cutoff: $300 \mathrm{~Hz}$ ) was applied. Continuous tracking of the head position relative to the MEG sensors allowed correction of head displacement during the breaks and before each file saving of a participant, using the fieldtrip toolbox (http:// fieldtrip.fcdonders.nl; Stolk et al., 2013).

\section{Behavioral analysis}

A "yes-no" model for independent observations was used to compute dprime (Green and Swets, 1966). Four classes of response are considered: (1) hit: a "yes" response when the target chunk is present in the digit sequence; (2) correct rejection: a "no" response when the target chunk is absent; (3) miss: a "no" response when the target chunk is present; and (4) false alarm: a "yes" response when the target chunk is absent. Nonparametric Wilcoxon signed-rank tests (twosided) were used to test differences in the mean dprime across conditions. The Bayes factor $B_{10}$ (Schönbrodt and Wagenmakers, 2018), which reflects the likelihood data arose from the alternative model, was computed using the software JASP (JASP Team, 2020; 10,000 samples) and default priors.

\section{MRI analysis}

The FieldTrip toolbox (http://fieldtrip.fcdonders.nl; Oostenveld et al., 2011) was used for the MRI and MEG data analyses. The standard Montreal Neurologic Institute (MNI) template brain was used for participants where an individual MRI was missing. Probabilistic tissue maps (cerebrospinal fluid gray and white matter) were constructed from the individual MRIs. Next, a single shell volume conduction model (Nolte, 2003) was applied to retrieve the physical relation between sensors and sources. Between the individual T1 MRI and the MNI template $\mathrm{T1}$ a linear warp transformation was computed. An 8-mm template grid, defined on the MNI template T1, was warped on the individual head space by inversely transforming it, based on the location of the coils during the MEG recording and the individual MRI. Next, based on the warped MNI grid and the probabilistic tissue map a forward model was computed, and applied for source reconstruction. This allowed aligning the grids of all participants to each other in MNI space for the across participants statistics.

\section{MEG preprocessing}

Line-noise was removed using bandstop filters (49.550.5, 99.5-100.5, two-pass; filter order 4) and the data were bandpass filtered off-line $(0.1-100 \mathrm{~Hz}$, Butterworth filter; filter order 4). A common semi-automatic artifact detection procedure was applied: for artifact rejection, the signal was filtered to identify muscular artifacts (bandpass: $110-140 \mathrm{~Hz}$ ) or jump artifacts (median filter) and znormalized per time point and sensor. The $z$ scores were averaged over sensors, to accumulate evidence for artifacts that occur across sensor. Trials that exceeded a predefined $z$ value (muscular artifacts, $z=15$; jumps, $z=30$ ) were rejected. Trials were the range (min-max difference) in any sensor exceeded a threshold (threshold $=0.75 \mathrm{e}-5$ ) were identified as containing slow artifacts and rejected. Down-sampling to $500 \mathrm{~Hz}$ was applied. The data were epoched (-3.5-5 s). Furthermore, when head movements exceeded a threshold $(5 \mathrm{~mm})$ a trial was rejected. Next, all blocks of recorded MEG data were concatenated. If high variance was detected at any sensor, the sensor was rejected. Finally, independent component analysis (infomax algorithm; Makeig et al., 1996) was used to remove eyeblink, eye-movement and heartbeat-related artifacts based on cumulative evidence from the component topography and time course.

\section{MEG source-level analysis}

In a first step, the data were epoched ( $0-5 \mathrm{~s})$. For the main analyses, only trials in which participants showed correct responses (i.e., hits and correct rejections) were selected. Next, the sensor-space measurements were projected and localized in source-space inside the brain volume (Van Veen et al., 1997) using linearly constrained minimum variance (LCMV) beamforming. A spatial filter was computed based on the individual leadfields for each participant and condition $(\lambda=10 \% ; 0.8-\mathrm{cm}$ grid). Next, all trials were epoched to the minimum stimulus duration in the corresponding condition (condition $1.8 \mathrm{~Hz}$ : $2.38 \mathrm{~s}$; condition $2.6 \mathrm{~Hz}: 1.68 \mathrm{~s}$ ).

\section{Cortical regions of interest (ROIs)}

The automated anatomic labeling atlas (AAL; TzourioMazoyer et al., 2002) was used to select the ROls as follows (Fig. 2): 


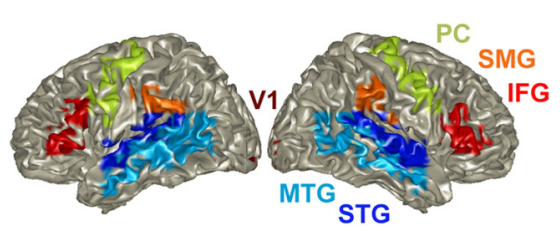

Figure 2. Cortical ROls. The automated AAL (Tzourio-Mazoyer et al., 2002) was used to select Cortical regions of interest (ROIs) in left and right STG, middle temporal gyrus (MTG), and speech-motor planning and integration areas (IFG, PC, SMG). $\mathrm{V} 1$ was used as control region. ROls are color coded.

- Superior temporal gyrus (STG; Temporal_sup_L/R): auditory association areas (Hickok and Poeppel, 2007; Binder et al., 2009)

- MTG (Temporal_Mid_L): implicated in processing word form and meaning

- IFG (Frontal_Inf_Tri_L/R): involved in speech-motor planning

- PC (Precentral_L/R), SMG (SupraMarginal_L/R): speechmotor integration

- Calcariane (Calcarine_L/R): primary visual cortex (as a control region)

We opted to omit Heschl's gyrus (primary auditory cortex area) from the list of ROls because of the very small number of voxels (three in the left, two in the right).

\section{Periodicity density function (PDF) within ROI}

We aim to determine whether the elicited brain signal measured at any given voxel within a specific ROI shows periodicity, and if so, to extract the frequency. Ultimately, we seek to characterize the density function of the periodicities across all voxels in the ROls of interest.

\section{The aggregated cross-correlation measure (XCOV) of periodicity}

To measure the neural response periodicity in individual voxels one could use one of several widely used measures, e.g., autocorrelation, where the first nontrivial peak indicates the period, or the intertrial phase coherence (ITPC), where the outcome would be the frequency distribution of the coherence function. Importantly, these measures build on the number of trials, $M$. The trial signals are noisy, both because of the SNR and because of the brain wave irregularity (which is why these methods average over trials). But what if $M$ is too small? Here we used a newly proposed measure, termed XCOV, to measure periodicity across $\mathrm{M}$ trials. Broadly, we suggest taking advantage of the fact that, for $M$ trials, we can generate about $\mathrm{M}^{2} / 2$ cross-correlation functions. Recall that, unlike autocorrelation, the first peak of a cross-correlation function does not indicate the period but rather the delay between the two signals. Therefore, we run each of the $\mathrm{M}^{2} / 2$ candidate pairs through a "match filter," which determines whether the corresponding two signals have a "zero" delay. Such a pair will have a cross-correlation function similar to that of an autocorrelation function, i.e., its peak is at zero and its earliest nontrivial peak is at the period. Only the pairs that pass the test are cross- correlated and aggregated. Obviously, the number of cross-correlation functions qualified for aggregation is between $\mathrm{M}$ and $\mathrm{M}^{2} / 2$, depending on how strict the match filter is. [For example, in the STG ROI, the mean number of trials over subjects for the "hit" response was $M=38$, with a mean number of pairs of 703 . The mean number of pairs that passed the test was 433 for the $1.8-\mathrm{Hz}$ condition and 378 for the 2.6- $\mathrm{Hz}$ condition, about one degree of order bigger than M. A similar trend was observed for all ROls.] We term the outcome of this measure as the XCOV function.

\section{PDF within a ROI}

Figure 3 details the analysis pipeline for deriving the PDF of the periodicities within a particular ROI. L voxels, $\mathrm{N}$ subjects, and $\mathrm{M}$ trials per subject are considered. First (data not shown), each brain signal is filtered to the frequency range of interest [low pass filter with cutoff frequency of $6 \mathrm{~Hz}$ for the (inside/outside) $\delta$ chunk rate analysis, and a bandpass filter with a [2-10] Hz frequency range, for $\theta$ (single digit rate) analysis]. [The filters were chosen with a bandwidth wider than the expected mean periodicities $(1.8$ and $2.6 \mathrm{~Hz}$ for $\delta, \sim 4 \mathrm{~Hz}$ for $\theta$ ), to let the XCOV analysis determine the periodicity PDFs without any bias.] Cross-correlations were computed using the filtered signals. Shown is the XCOV function at the $\mathrm{i}$-th voxel, for the $\mathrm{j}$-th subject, obtained by aggregating $\mathrm{K}$ cross-correlation functions. (Note that as a cross-correlation function, XCOV is computed against time-lags; the abscissa here shows the time-lag inverse, in frequency, hence going from right to left). The particular XCOV function shown in Figure 3 has a single peak at $1.76 \mathrm{~Hz}$ but note that, in general, an XCOV may have more than one local peak. Next, the location of the prominent peaks is extracted, with the number of prominent peaks as a parameter. (The prominence of a peak measures how much the peak stands out because of its intrinsic height and its location relative to other peaks in the range of interest.) In our analysis one prominent peak per XCOV is considered. Hence, for $L$ voxels and $\mathrm{N}$ subjects, a maximum of $\mathrm{L} \times \mathrm{N}$ data points are available to construct a histogram, from which only those inside the frequency range of interest are used, and the resulting histogram is normalized to $L \times N$. A third order Gaussian mixture model (GMM) that fits the histogram is the desired PDF. The "goodness" of the periodicity is quantified by in terms of $P$, the percentage of datapoints inside the frequency range of interest with respect to the total number of datapoints $(\mathrm{L} \times \mathrm{N})$, and the mean $\mu$ and variance $\sigma$ of the prominent Gaussian component of a third order GMM. (The total number of data points is shown in the inset of each entry.)

\section{Software accessibility statement}

Analysis code will be made available on request.

\section{Results}

\section{Behavioral results}

Dprime scores were the highest in the $1.8-\mathrm{Hz}$ condition (mean $=2.19, \mathrm{SD}=0.4$; Fig. 4), i.e., when the chunk rate is inside the $\delta$ frequency range. Lower dprime scores were 


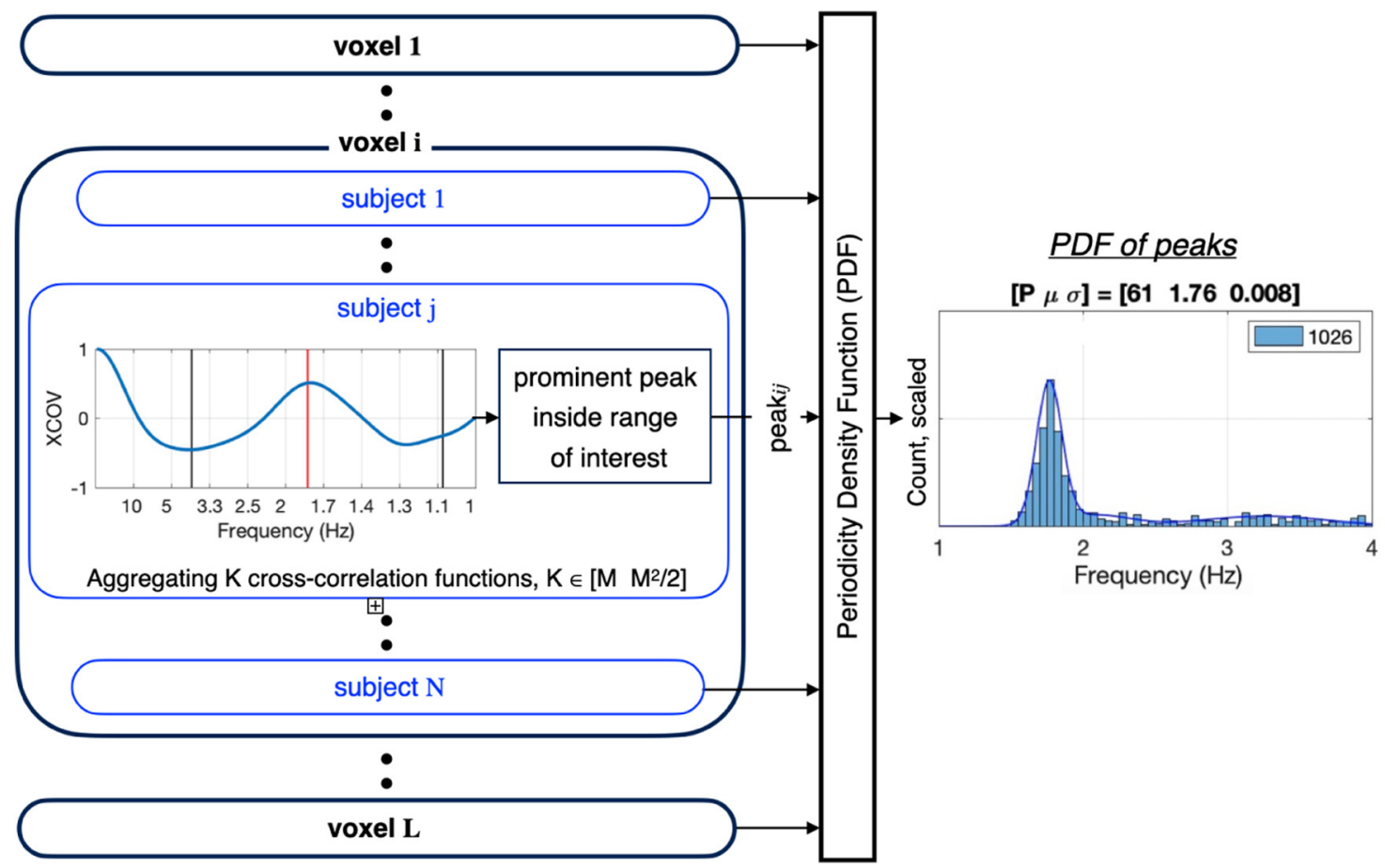

Figure 3. Analysis pipeline for deriving the PDF within a particular ROI. Shown is the resulting periodicity density function (PDF) for a given condition (say, the 1.8- $\mathrm{Hz}$ condition), a given response class (say, hit), and a given region of interest (ROI; say, STG). L voxels, $\mathrm{N}$ subjects, and $\mathrm{M}$ trials per subject are considered. For the i-th voxel and the j-th subject, periodicity is computed using a newly proposed measure method termed XCOV. First (data not shown), each brain signal is filtered to the frequency range of interest. Cross-correlations were computed using the filtered signals. Note that as a cross-correlation function, XCOV is computed against time-lags; the abscissa here shows the time-lag inverse, in frequency, hence going from right to left. The PDF is derived by (1) forming a histogram of the XCOV peak locations inside the frequency range of interest; (2) normalizing the histogram to $L \times N$, the total number of data points; and (3) building a third order Gaussian Mixture Model (GMM) that fits the histogram. The GMM model is the desired PDF. The goodness of the PDF is quantified by in terms of $p$ value, the percentage of datapoints inside the frequency range of interest with respect to the total number of datapoints $(\mathrm{L} \times \mathrm{N})$; and the mean $\mu$ and variance $\sigma$ of the prominent Gaussian component of a third order GMM. (The total number of data points is shown in the inset of each entry.)

registered in the 2.6- $\mathrm{Hz}$ condition (mean $=1.74, \mathrm{SD}=0.42$ ), when the chunk rate was just at the outside edge of the $\delta$ range. The difference in scores was significant $(1.8-\mathrm{Hz}$ condition vs $\left.2.6 \mathrm{~Hz}: W=177, p<0.001, r=0.863 ; \mathrm{BF}_{10}=199.6\right)$.

\section{PDF of elicited brain waves}

We used the XCOV of periodicity across $M$ trials to determine whether the elicited brain signal measured at any given voxel within a specific $\mathrm{ROI}$ shows periodicity, and if so, to extract the frequency. Then, we derived the PDF of the periodicities across all voxels in the ROls of interest (Fig. 2). The goodness of the periodicity is quantified by in terms of $P$, the percentage of datapoints inside the frequency range of interest with respect to the total number of datapoints ( $L$ voxels $\times N$ subjects), and the mean $\mu$ and variance $\sigma$ of the prominent Gaussian component of a third order GMM. Figure 5 shows the periodicity PDFs in the [1 4] Hz frequency range for the STG ROI in the left hemisphere. For the 1.8- $\mathrm{Hz}$ condition, a strong periodic response at $\sim 1.8 \mathrm{~Hz}$ was recorded for the hits and correct rejections, with the $\mathrm{P}$ over $50 \%$. Much weaker presence of periodicity was recorded for the misses and false alarms. A similar trend is shown for the $2.6-\mathrm{Hz}$ condition, albeit with much weaker periodicity compared with the $1.8-\mathrm{Hz}$ condition, and with a smaller $\mathrm{P}$ (of below $30 \%$ ). Notice that, across chunk conditions, the PDF patterns for hits and correct rejections are similar, as are the patterns for misses and false alarms. Such similarities were observed for all ROls. Therefore, in presenting the rest of the data, the hits and correct rejections are combined to indicate correct responses, and the misses and false alarms are as erroneous responses.

In the following figures, the data are presented as follows. Each figure contains $6 \times 2$ entries organized in six rows (ROls) and two columns (chunking conditions). Each entry shows the periodicity PDF, and the goodness of the periodicity is quantified in terms of $\mathrm{P}, \mu$ and $\sigma$. In some selected entries, the upper left corner shows the KullbackLeibler divergence (KLD) of the entry's PDF with respect to a reference PDF defined in the respective figure caption. Finally, in some entries, no $\mu$ and $\sigma$ values are present. This is so because of a failure of the third order GMM to converge because of the small $p$ value.

Figure $6 A, B$ show the elicited responses in the [1 4$] \mathrm{Hz}$ frequency band for correct responses (i.e., hits and correct rejections combined), and erroneous responses (i.e., misses and false alarms combined), respectively. We 


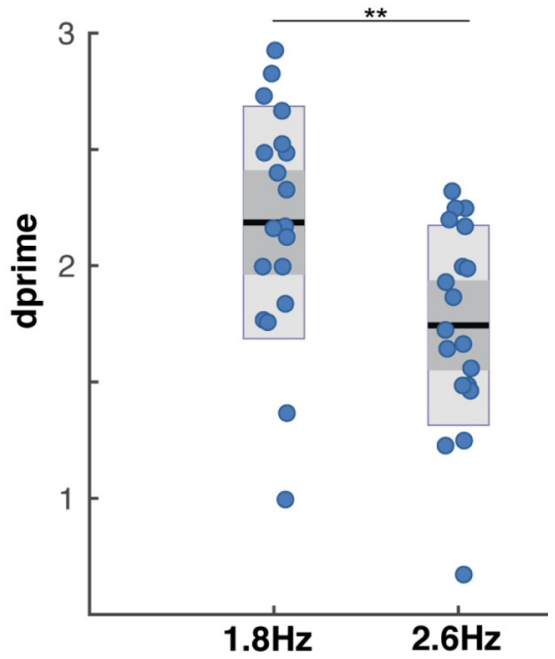

Figure 4. Behavioral performance in the digit retrieval task. Dprime values are displayed, as measure of performance accuracy, separately for each condition. Blue dots indicate individual dprime scores, black lines indicate the mean dprime scores, dark gray bars indicate the \pm 1 SEM and light gray bars the confidence interval. Significance is indicated by ${ }^{* *}(p<0.01)$. The performance was higher in the $1.8-\mathrm{Hz}$ acoustical chunk (inside $\delta$ chunking), compared with the $2.6-\mathrm{Hz}$ acoustical chunk condition (outside of $\delta$; replicating findings in Ghitza (2017)).

term these elicited responses $\delta$ responses. For correct responses in the $1.8-\mathrm{Hz}$ condition a strong periodicity presence at $\sim 1.8 \mathrm{~Hz}$ is recorded. A similar pattern is shown for the $2.6-\mathrm{Hz}$ condition, albeit with much weaker periodicity presence compared with the $1.8 \mathrm{-Hz}$ condition (lower $p$ value and wider $\sigma$ ). For erroneous responses, for all ROls, no presence of periodicities is recorded, for any condition. More specifically: for correct responses, in the chunked conditions, the auditory association ROI (STG) shows a compelling periodicity presence at $1.8 \mathrm{~Hz}$ in the $1.8 \mathrm{-Hz}$ condition and a weaker presence at $2.6 \mathrm{~Hz}$ in the $2.6-\mathrm{Hz}$ condition. At the middle temporal ROI (MTG), periodicity exists for the chunked conditions, albeit with $1.8 \mathrm{~Hz}$ periodicity stronger than that of $2.6 \mathrm{~Hz}$. Similar patterns are observed in the speech-motor planning and integration ROls (IFG, SMG, PC), whereas periodicity is present at $1.8 \mathrm{~Hz}$, and is absent in the $2.6-\mathrm{Hz}$ condition. Note that in the visual ROI (calcarine), $\delta$ periodicities are absent for all conditions. Finally, the $1.8-\mathrm{Hz}$ condition column of Figure $6 \mathrm{~A}$ also shows the KLD for all ROls, with respect to the STG $\mathrm{ROI}$ (highlighted in red). The KLD values suggest similar patterns of elicited $\delta$ periodicities observed in the temporal brain regions (STG and MTG ROIs, with KLD value of 0.15 for MTG), and the frontal motor and temporal-parietal regions (IFG, SMG and PC ROls, with KLD values of 0.15 , 0.25 , and 0.11 , respectively).

Furthermore, we compared the elicited $\delta$ responses in all ROIs in the left versus the right hemispheres for correct responses. Similar periodicity PDFs are observed for all ROIs in all chunking conditions. The KLD was calculated for each ROI in the Right hemisphere against the corresponding left ROI. The KLD values show a closer similarity between the periodicity PDFs of the left and right hemisphere of the temporal brain regions (STG and MTG, with KLD values of 0.1 and 0.11 , respectively). In contrast, in the frontal motor and temporal-parietal regions periodicities were more prominent in the left compared with the right hemisphere (IFG, SMG, and PC, with KLD values of $0.28,0.15$, and 0.47 , respectively).

Figure $7 A, B$ show the elicited responses in the [2 6] $\mathrm{Hz}$ frequency band for the correct and erroneous responses, respectively, for ROls in the left hemisphere. We term responses in this frequency band $\theta$ responses. For the correct behavioral responses, strong $\theta$ was elicited in all ROls and for all chunking conditions. Such elicited neural response patterns reflect the single digit presentation rate. Two observations are noteworthy, the bimodal characteristic of the PDFs for all chunking conditions, in particular for the $1.8-\mathrm{Hz}$ chunking condition, and the strong, unexpected, $\theta$ periodicity presence in the calcarine $\mathrm{ROI}$. For the erroneous responses, a weaker more dispersed periodicity presence was observed. Finally, for the correct responses, the periodicity PDFs were similar in shape across conditions, as was quantified by the KLD values comparing the periodicity PDFs in the $1.8-\mathrm{Hz}$ condition with respect to the 2.6- $\mathrm{Hz}$ condition (KLD values between 0.13 and 0.2 across ROls). The similarity of the PDFs across chunking conditions confirms that the decoding time at the digit level was sufficient across conditions.

\section{Correspondence between behavioral data and electrophysiological data}

Figure 8 quantifies the correspondence between the elicited $\delta$ periodicity patterns and the behavioral data. Shown are the third order GMMs computed for the correct responses in the left hemisphere and the two stimulus conditions. Unlike Figure $6 A$, which shows PDF in terms of scaled periodicity count, shown here are the actual probability densities (with the $\int p(x) d x=1$ ). The title of each panel shows three measures: (1) [dprime $\sigma$ ], the behavioral performance indicated by mean dprime values and the variance across subjects; (2) [Bias $\sigma$ ], the average of the absolute difference (termed Bias) between the mean of the prominent Gaussian component of the GMM and the acoustic chunk rate, and the variance across the ROls; and (3) $[P \sigma]$, the average $p$ value and the variance across the ROls. Two observations are noteworthy. First, the tightness of the PDFs in the $1.8-\mathrm{Hz}$ condition as reflected in the high probability value at the periodicity frequency, compared with the pseudo-uniform shape of the PDFs in the 2.6- $\mathrm{Hz}$ condition. And second, the decrease in dprime accompanies the increase in Bias and the decrease in $P$. These data support the hypothesis that perceptual chunking at the time scale of phrase is derived by acoustic-driven $\delta$ oscillators.

\section{Discussion}

In this study we adopted a reductionist approach to test, in electrophysiological terms, the hypothesis that the speech decoding process at the phrasal time scale is guided by a flexible, acoustic-driven neuronal $\delta$ oscillator locked to phrase-size acoustic cues (Ghitza, 2017). The proposal suggests an analog role of a $\delta$ oscillator, at the 

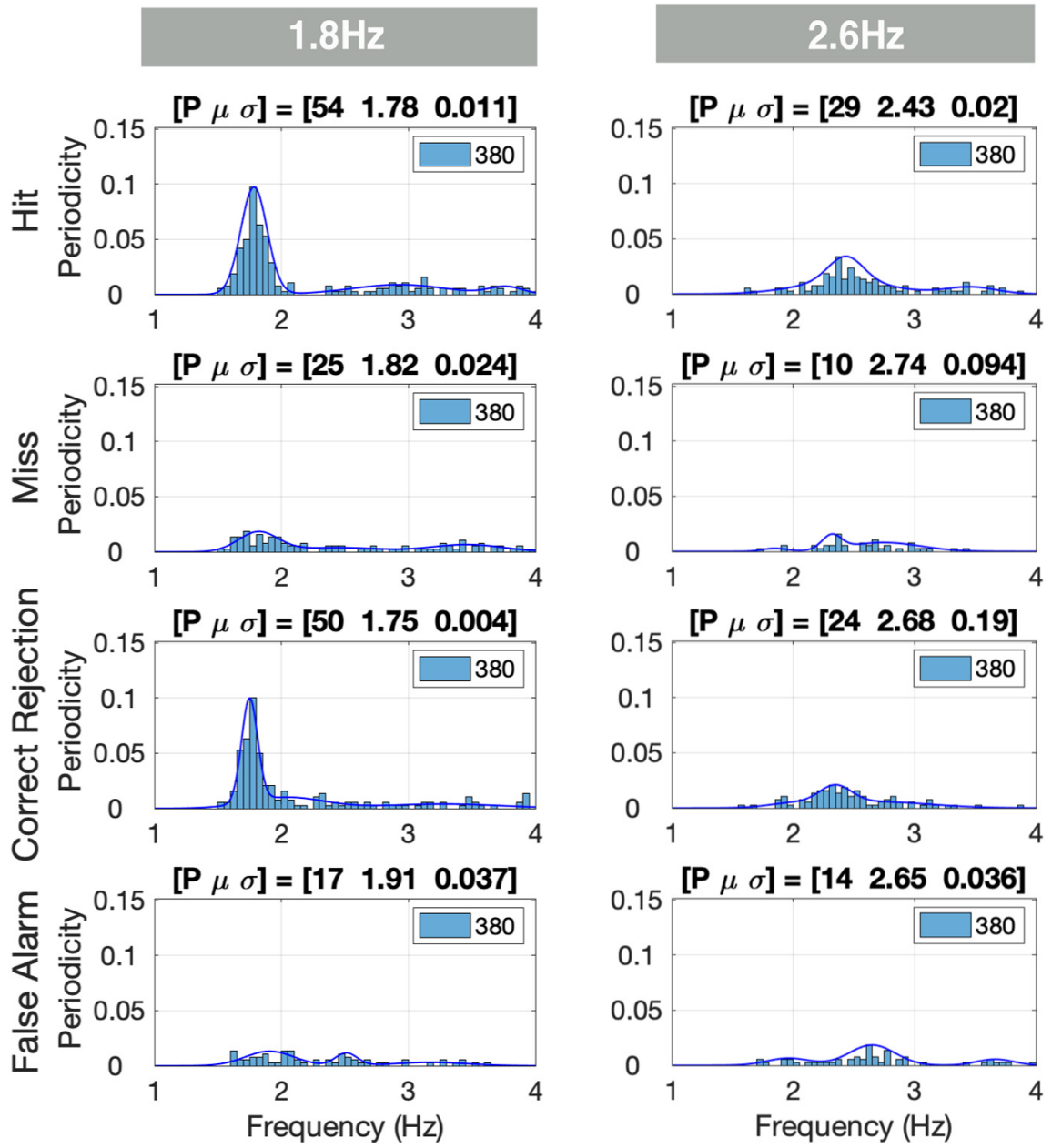

Figure 5. PDFs of $\delta$ periodicities per response class. The periodicity density functions (PDFs) are displayed for the left hemisphere STG region of interest (ROI). The number of voxels in this $\mathrm{ROI}$ is 20 and the number of participants 19 . Per voxel and subject, one

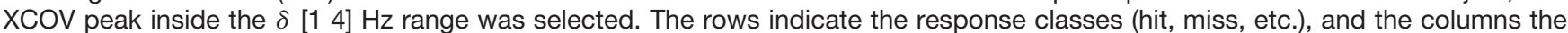
chunking conditions. Each entry shows the histogram (with the periodicity count scaled to $L \times N$ ), and the corresponding PDF. The inset of each entry shows the total number of data points $\mathrm{L} \times \mathrm{N}$ analyzed ( 20 voxels $\times 19$ subjects $=380$ incidences). The goodness of the PDF is quantified in terms of the percentage ( $p$ value) of datapoints inside the frequency range of interest with respect to the total number of datapoints, and the mean $\mu$ and variance $\sigma$ of the prominent Gaussian component of a third order GMM. For the $1.8-\mathrm{Hz}$ condition, a strong periodicity presence at $1.8 \mathrm{~Hz}$ was recorded for the hit and correct rejection responses, with $\mathrm{P}$ over $50 \%$. A much weaker presence was recorded for the miss and false alarm responses. A similar trend is shown for the 2.6- $\mathrm{Hz}$ condition, albeit with much weaker periodicity presence compared with the 1.8- $\mathrm{Hz}$ condition, and a smaller number of datapoints (P of below $30 \%$ ).

phrasal time scale, to the role played by neuronal $\theta$-band oscillations at the syllabic time scale. The study is reductionist in the sense that it is confined to the perceptual chunking of digits sequences, where the digits in the sequence are grouped into phrase-size chunks. We collected, concurrently, behavioral and MEG data during a digit retrieval task, in which the digit sequences were either presented with an acoustic chunk pattern inside or outside of the $\delta$ range. Stimuli with a chunk rate inside the $\delta$ range elicited considerable neuronal periodicity at the chunk rate in STG, MTG ROls and IFG, SMG, and PC ROIs. Critically, this pattern of detected periodicities was directly related to correct behavioral responses. In contrast, stimuli with a chunk rate outside of the $\delta$ range elicited weak periodicity, aligned with observed declines in behavioral performance. In the calcarine ROI (early visual cortex), considered a "control area" for our analyses, no periodicities at the chunk rate were elicited.

\section{Presence of $\delta$ periodicities in the auditory pathway}

How should these activity patterns of neuronal $\delta$ and $\theta$ periodicities, be interpreted? In the temporal cortex (STG and MTG), robust periodicities were recorded mainly by stimuli with a chunk rate inside the $\delta$ range, and only for correct behavioral responses. Periodicities in these brain areas were present even for acoustic chunk rates at the edge of the $\delta$ range, albeit considerably weaker. A similar pattern of periodicities was observed in the speech-motor planning and integration areas (IFG, SMG, and PC), where periodicities were absent for acoustic chunk rates outside the $\delta$ range. Note that the observed lack of hemispheric lateralization in auditory cortex in our study is in line with 
A

\section{$1.8 \mathrm{~Hz}$}

Correct Response
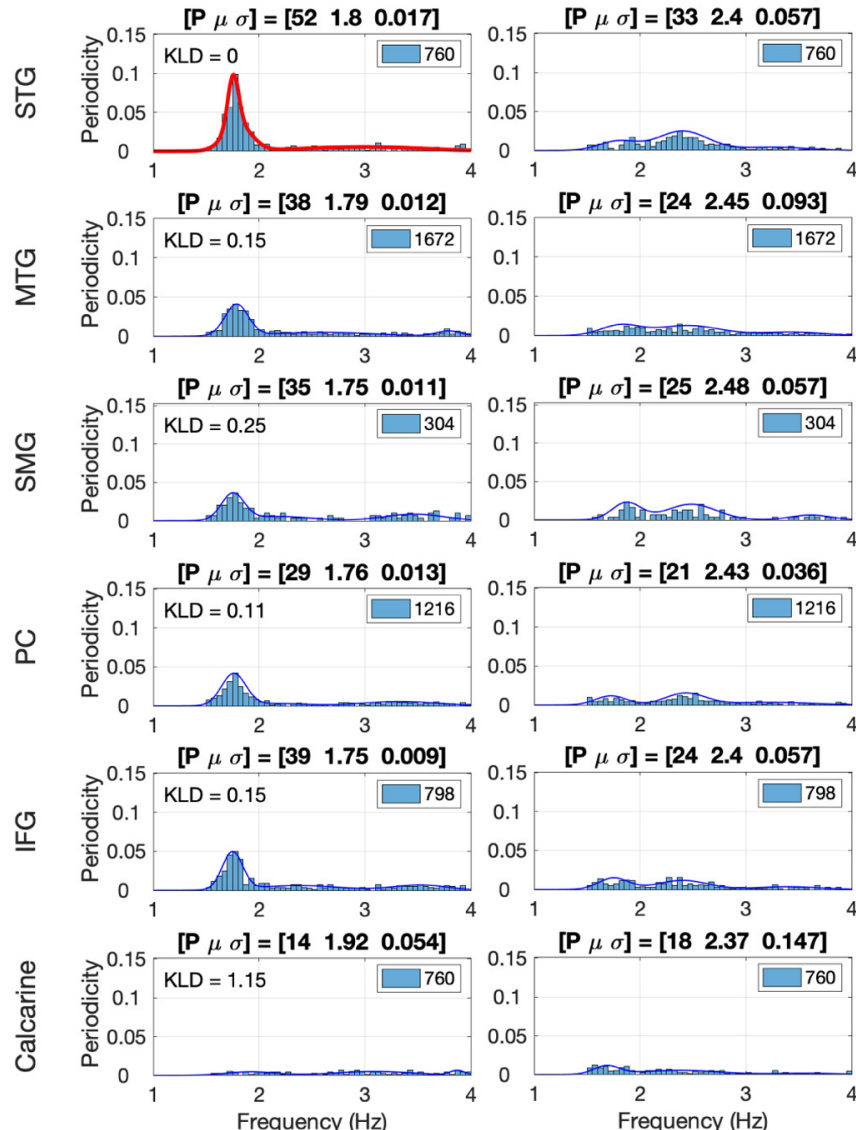

B

\section{Erroneous Response}

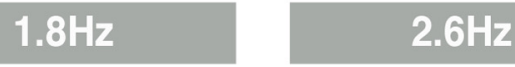

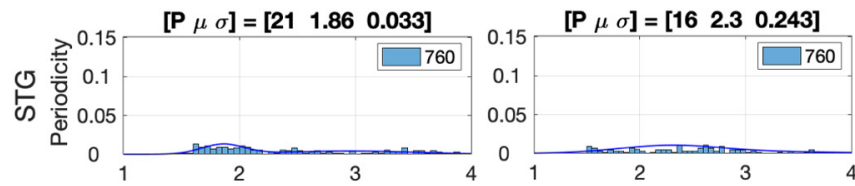
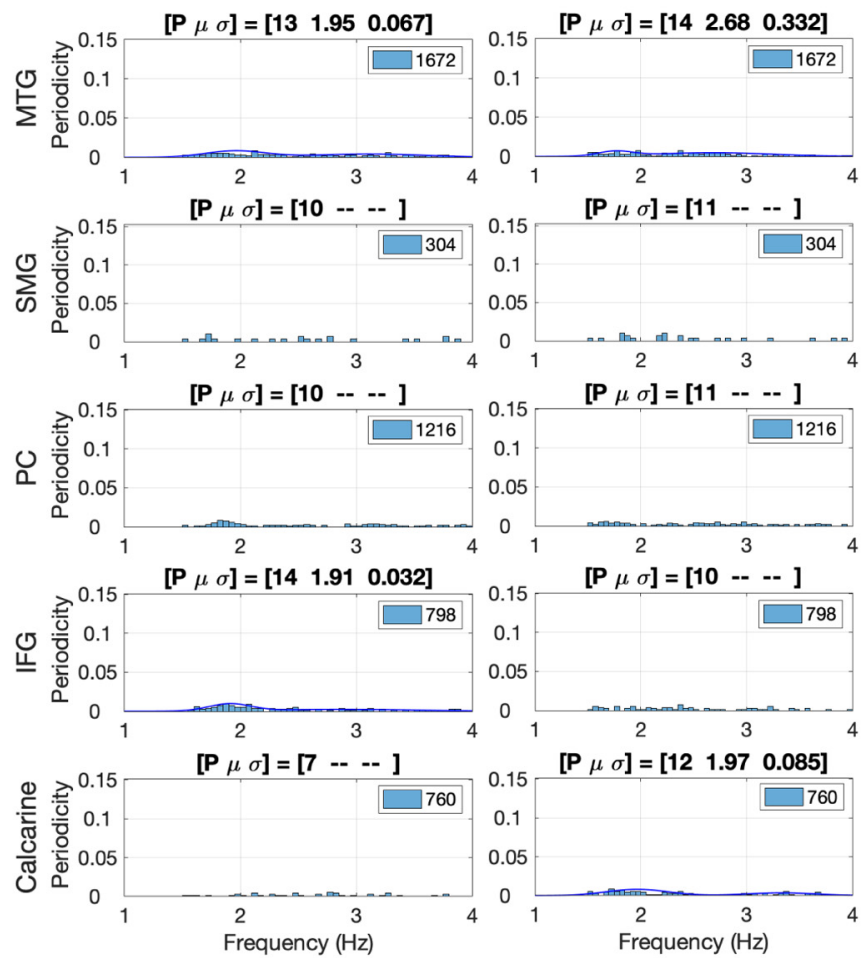

Figure 6. $\delta$ Periodicities for correct and erroneous responses in the left hemisphere. A, Periodicities for correct responses: rows indicate the ROIs and columns the chunking conditions. Each entry shows the histogram (with the periodicity count scaled to the $L \times$ $\mathrm{N}$ ), and the PDF, quantified in terms of the percentage ( $p$ value) of datapoints inside the frequency range of interest with respect to the total number of datapoints $(\mathrm{L} \times \mathrm{N}$, see inset). For the 1.8- $\mathrm{Hz}$ condition, a strong periodicity presence at $\sim 1.8 \mathrm{~Hz}$ is recorded. $\mathrm{A}$ similar trend is shown for the $2.6-\mathrm{Hz}$ condition, albeit with much weaker periodicity presence compared with the 1.8- $\mathrm{Hz}$ condition. The 1.8- $\mathrm{Hz}$ condition column shows the KLD computed for this condition at all ROIs, with respect to the STG ROI highlighted in red (upper left corner of the ROIs). The KLD values suggest similar patterns of elicited $\delta$ periodicities in the temporal brain areas (STG and MTG ROIs), and in the frontal motor and temporal parietal areas (IFG, SMG, and PC ROIs). B, Periodicities for erroneous responses: no presence of periodicities is recorded for any condition.

previous reports on bilateral $\theta / \delta$ activity elicited to more complex speech stimuli (Assaneo et al., 2019; Flinker et al., 2019). Interestingly, in contrast to the temporal brain areas, in the speech-motor planning and integration areas more divergence between the left and right hemisphere was observed, with more prominent $\delta$ periodicities in the left hemisphere. The left hemisphere more tightly followed the chunking rate compared with the right. These findings suggest an important role for superior and middle temporal and speech-motor planning and integration areas in chunking at the phrasal scale. Importantly, and quite remarkably, the $\delta$-band activity in these areas was fully aligned with behavioral performance (i.e., $\delta$ activity was only elicited in correct, but not in erroneous responses). Previously, EEG studies showed $\delta$ in bilateral middle and superior temporal areas (also fusiform gyrus; Bonhage et al., 2017) and at frontotemporal sites (Boucher et al., 2019) was related to chunking during phrase and sentence processing. $\delta$ Might reflect the chunking of ordered sensorimotor events as articulated sound, rather than syntactic phrasal/sentential processing directly (Boucher et al., 2019). Furthermore, Keitel et al. (2018) and Morillon et al. (2019) recently proposed that $\delta$ oscillations in the motor cortex are involved in temporal predictions, affecting speech processing in the auditory cortex at a phrasal scale (for a predictive account of $\delta$ see also Breska and Deouell, 2017; Daume et al., 2021; or a statistical learning account, see Henin et al., 2021). A possible interpretation of their findings through the lens of our results is that acoustic-driven segmentation of phrase-size chunks takes place in STG, and the recorded behavioral performance with respect to chunk rate is a consequence of the 
A

\section{Correct Response}

\section{$1.8 \mathrm{~Hz}$}
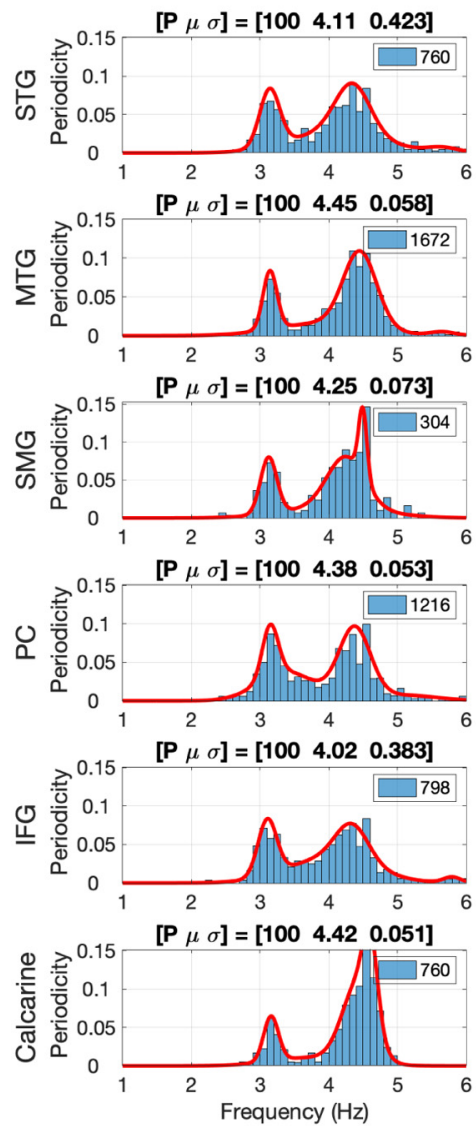

2.6 Hz
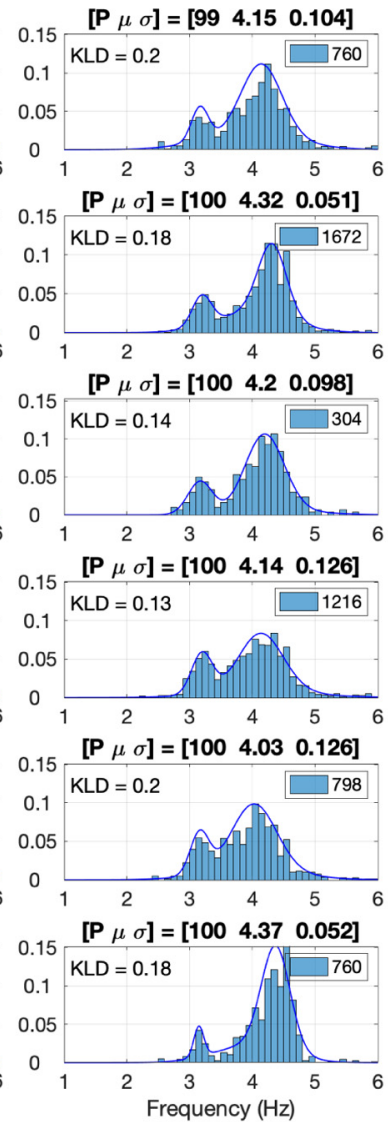

B

Erroneous Response

$1.8 \mathrm{~Hz}$

2.6 $\mathrm{Hz}$
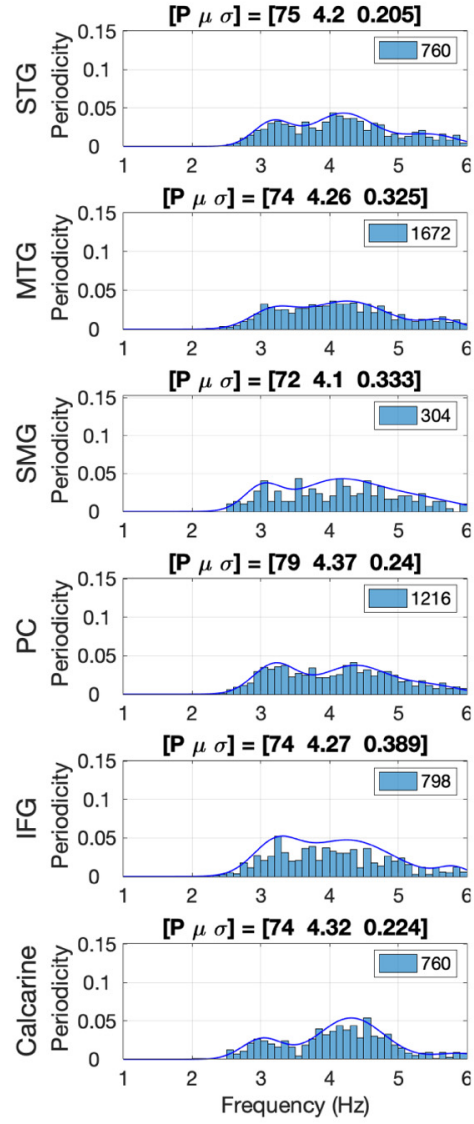
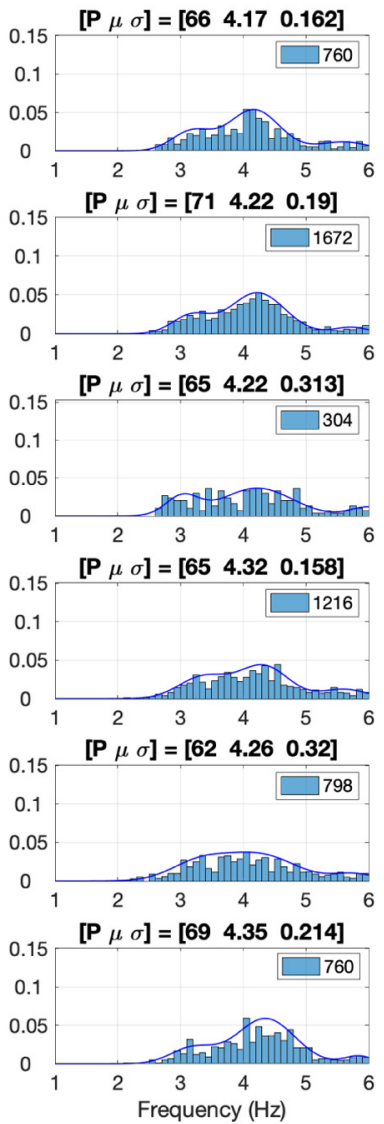

Figure 7. $\theta$ Periodicities for correct and erroneous responses in the left hemisphere. A, Periodicities for correct responses: strong $\theta$ periodicities were present in all regions of interest (ROIs) and for all chunking conditions. Such elicited neural response patterns reflect the single digit presentation rate. The histograms are scaled to $L \times N$ (see inset). The periodicity density functions (PDFs) are similar in shape across conditions, as is quantified by the KLD values comparing the PDFs in the 1.8-Hz condition with respect to the 2.6- $\mathrm{Hz}$ condition. $\boldsymbol{B}$, Periodicities for erroneous responses: a weaker more dispersed presence of $\theta$ periodicities is recorded for all conditions (lower $p$ value and wider $\sigma$ ).

goodness of segmentation. When the chunk rate is inside the $\delta$ band, successful segmentation results in $\delta$ activities in speech-motor integration areas (SMG, PC, IFG) that may reflect decoding processes and possibly auditorymotor mapping related processes (Park et al., 2015). In contrast, chunk rates outside of the $\delta$ band might result in bad segmentation in STG, and in turn suppressed periodicities in speech-motor integration areas (SMG, PC, IFG) because of unreliable decoding and audio-motor mapping. This interpretation is in line with another study (Donhauser and Baillet, 2020) that reports strong $\delta$ activity in STG when the speech input was "informative," which may be the consequence of appropriate segmentation.

It could be argued that one cannot draw a conclusive relationship between "chunking" and the neural periodicity in the $\delta$ range. In particular, the drop in intelligibility for the $2.6-\mathrm{Hz}$ condition may be because of the fact that the silent gaps in-between the two-digit chunks are shorter.
This argument raises three points that merit discussion. First, a question arises whether or not a $2.6 \mathrm{~Hz}$ rhythm in the acoustics is present at the cochlear output level. Figure $9 A$ shows a simulation of the cochlear modulation spectrum (Jepsen et al., 2008) for a $1.8 \mathrm{~Hz}$ (left) and a $2.6 \mathrm{~Hz}$ (right) stimuli, taken at a characteristic frequency of $426 \mathrm{~Hz}$ (this cochlear place was selected at random, for demonstration). A robust modulation presence is observed for both stimuli, at their respective acoustic input rhythm. Second, it could be argued that the shorter silent gaps result in weaker acoustic cues for chunking. Recalling that neural activity in primary auditory cortex represents sensory representations of the acoustics with a minimal information loss (Nourski et al., 2009), a weakening in acoustic cues should be reflected in terms of a weaker periodicity presence at primary cortex (e.g., the Heschl's gyrus). As mentioned earlier (Fig. 2), we opted to omit the Heschl's gyrus from our rigorous periodicity analysis because of the small number of voxels present (three 

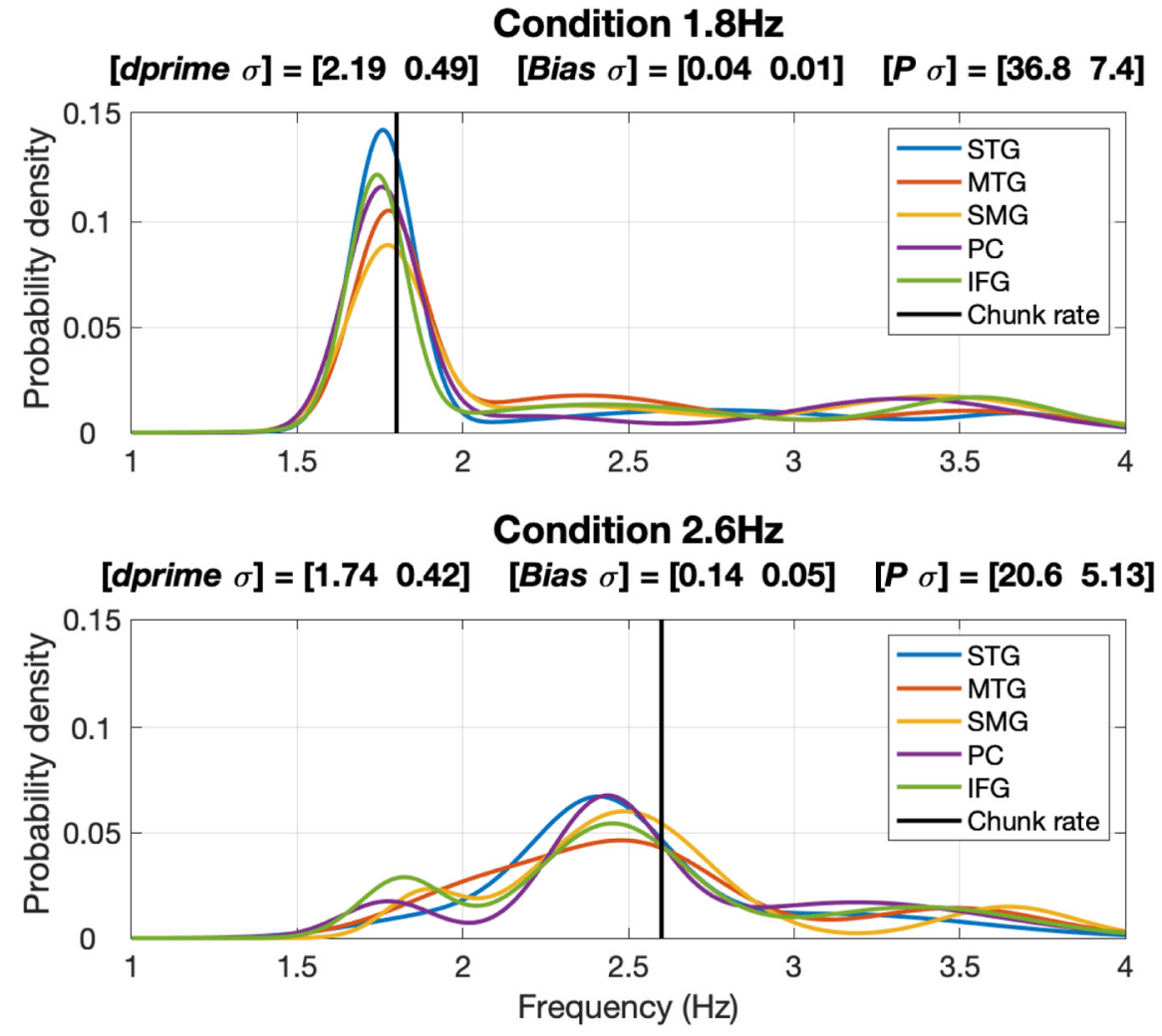

Figure 8. Correspondence between behavioral data and electrophysiological data. Shown are the third order Gaussian Mixture Models (GMMs) of correct responses in the left hemisphere. Unlike Figure 6A, which shows the periodicity density function (PDF) scaled to $\mathrm{P}$ (the percentage of datapoints inside the frequency range of interest with respect to the total number of datapoints), shown here are the actual probability densities (with the $\int p(x) d x=1$ ). The title of each panel shows three measures: (1) [dprime $\sigma$ ], the behavioral performance; (2) [Bias $\sigma$ ], the average of the absolute difference between the mean of the prominent Gaussian component of the GMM and the driving acoustic chunk rate, and variance across the ROls; and (3) [ $\sigma]$, the average $p$ value (defined in Fig. 6) and variance across the regions of interest (ROIs). Note the tightness of the PDFs in the 1.8- $\mathrm{Hz}$ condition compared with the pseudo-uniform shape of the PDFs in the $2.6-\mathrm{Hz}$ condition, and the correlation between the decrease in dprime and the increase in Bias and the decrease in $p$.

in the left, two in the right). Figure $9 B$ shows the XCOV periodicity PDF for all five available voxels, for correct and erroneous responses combined. Keeping in mind the concern over the validity of the results because of the limited number of voxels, we observe a strong periodicity presence for both chunking conditions at their respective chunk rates, suggesting no weakening of the acoustic cues for chunking. In contrast, as early as at the STG level we observe strong periodicities only for chunk rates inside the $\delta$ frequency range (Fig. 6). The findings suggest that the neuronal circuitry of the $\delta$ oscillator resides at the STG level and constrains prosodic chunking. Third, it could also be argued that the shorter silent gaps result in an insufficient decoding time at the single digit level. However, our data show that this is not the case, as at the digit level, for all chunking conditions and at all ROls, strong $\theta$ periodicities (at the single digit rate) were elicited regardless of the level of behavioral chunking performance. Thus, the drop in performance for the $2.6-\mathrm{Hz}$ condition, with a chunk rate just outside the $\delta$ frequency range, is because of the lack of decoding time at the chunk level but not because of digit decoding time. Recall that for both the $2.6-\mathrm{Hz}$ and the $1.8-\mathrm{Hz}$ stimuli, the two-digit chunks themselves have an identical time-compressed acoustics; the only difference is the duration of the silent gaps between the chunks (Fig. 1). Performance is recovered by bringing the chunk rate back inside the $\delta$ range, hence providing the extra decoding time needed. As a whole, therefore, our data suggest that segmentation of phrase-sized chunks is realized by neuronal $\delta$ oscillators, and that the chunk's decoding time is determined by $\delta$, in analogy to the role of $\theta$ in determining the decoding time at the syllable level (Ghitza, 2014).

\section{Presence of $\theta$ periodicities in all chunking conditions}

Our data show strong $\theta$ periodicities in all ROls and for all chunking conditions. Such elicited neural response patterns reflect the single digit presentation rate. A bimodal characteristic of the PDFs is observed for all chunking conditions, but in particular for the $1.8 \mathrm{-Hz}$ condition. The bimodality arises from the acoustic properties of the stimuli. Consider, for example, the stimulus shown in Figure 1. Three intradigit durations can be identified: (1) the duration between the onset of the first digit of a chunk and the first digit in the following chunk, which gives rise to the chunking rate; (2) the duration between the onset of 

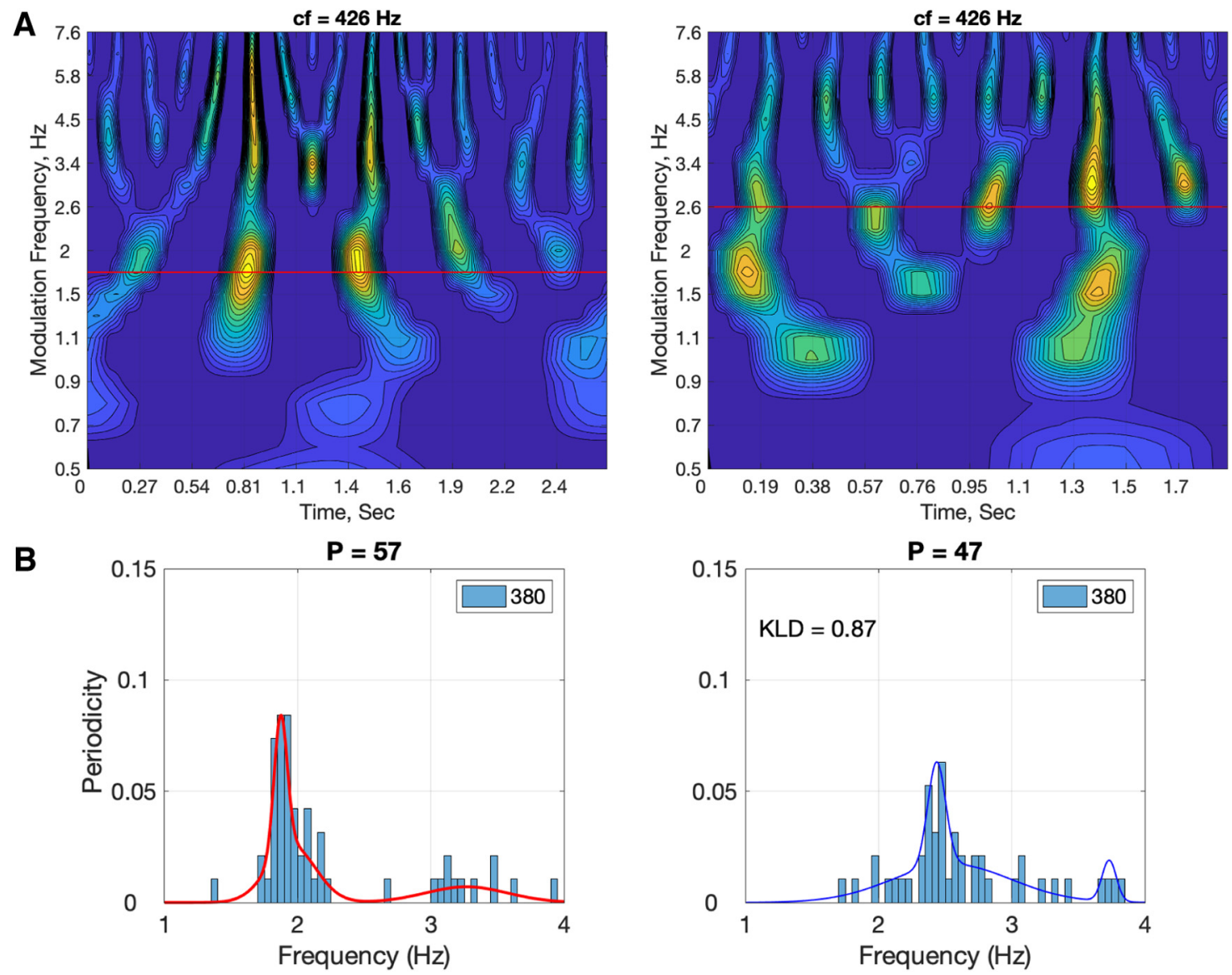

Figure 9. Cochlear modulation spectrum $(\boldsymbol{A})$ and $\delta$ periodicities at Heschl's gyrus $(\boldsymbol{B})$ for the digit-sequence stimuli shown in Figure 1. $\boldsymbol{A}$, Cochlear output in terms of cochlear modulation spectrum (Jepsen et al., 2008). Shown are modulation spectra of the digit-sequence stimuli shown in Figure 1, for the 1.8- $\mathrm{Hz}$ stimulus (left) and for the 2.6- $\mathrm{Hz}$ stimulus (right). The modulation spectra shown are snapshots at the cochlear characteristic frequency (CF) of $426 \mathrm{~Hz}$. Abscissae represent time (duration of 2.7 and $1.9 \mathrm{~s}$, for the $1.8-$ and the $2.6-\mathrm{Hz}$ stimuli, respectively) and the ordinate represents the modulation frequencies $(0.5 \mathrm{Ho} 7.6 \mathrm{~Hz})$. Note the strong presence of modulations at $1.8 \mathrm{~Hz}$ for the $1.8-\mathrm{Hz}$ stimulus and at $2.6 \mathrm{~Hz}$ for the $2.6-\mathrm{Hz}$ stimulus. (B) $\delta$ Periodicities at Heschl's gyrus ROI for the correct and erroneous responses, combined. Note that the total number of datapoints is 380: the number of voxels (left and right combined) is 5 , the number of participants 19 , and the number of response conditions (correct and erroneous) is 4 . The KLD value of the $2.6-\mathrm{Hz}$ probability density function (shifted to $1.8 \mathrm{~Hz}$ ) with respect to the $1.8-\mathrm{Hz}$ probability density function is 0.87 . Keeping in mind the concern over the validity of the results because of the limited number of voxels, the strong periodicity presence for both chunking conditions suggest that the diminished periodicity for the $2.6-\mathrm{Hz}$ condition is because of neuronal circuitry characteristics at the STG level and not because of weakening of acoustic cues for chunking.

the first digit and onset of the second digit in a chunk; and (3) the duration between the onset of the second digit in a chunk and the onset of the first digit in the following chunk. This plurality in intradigit durations give rise to a bimodal duration distribution with a skewness determined by the prescribed chunking rate. The skewness is accentuated, in particular, in our 1.8- $\mathrm{Hz}$ stimuli. The bimodal nature in the acoustics drives the elicited neural response seen in our data (Fig. 7A).

\section{Oscillations versus evoked responses}

Our data show strong $\delta$ cortical periodicities while listening to the $1.8 \mathrm{~Hz}$ chunked stimuli. Are these brain waves generated by a neuronal oscillator locked to the acoustic chunk rhythm or do they reflect the evoked response to the corresponding acoustic cues? The answer to this question at the syllabic level has been difficult to determine, because the impulse response of the neuronal circuitry to discrete acoustic cues associated with syllables (e.g., acoustic edges, vocalic nuclei) corresponds, in duration, to the $\theta$-cycle range (about [125 330] ms). Doelling et al. (2019) addressed this conundrum by generating simulated outputs of an oscillator model and of an evoked response model, and comparing the quantitative predictions of phase lag patterns generated by the two models against recorded MEG data. They showed that, compared with the evoked response model, a model that includes oscillatory dynamics better predicted the MEG data. Our data provides additional support for the oscillator interpretation. Can the observed, robust periodic responses to a $1.8 \mathrm{~Hz}$ chunked stimulus reflect evoked responses elicited by discrete acoustic cues at the phrase time scale? Indeed, steady-state evoked responses to 
slow dynamics have been observed in both visual and auditory sensory regions (Capilla et al., 2011; Wang et al., 2011). However, only a model of oscillatory dynamics can explain the fact that neural response at the $\delta$ range is only present when the acoustic chunk rate is inside, but is absent for rates outside the $\delta$ range.

\section{Generalizability of the neuronal chunking mechanism Scaling up to real speech}

The studies discussed above (Meyer et al., 2017; Bonhage et al., 2017; Keitel et al., 2018; Boucher et al., 2019; Morillon et al., 2019) suggest a presence of $\delta$ brain waves in phrasal chunking for continuous speech, beyond the digit retrieval paradigm used here. Extending our results to naturalistic speech has important implications for what would constitute optimally sized acoustic chunks for the sentential decoding, or parsing, process. If the information "bound" within windows of roughly a $\delta$ cycle are integrated as phrases (intonation phrases and perhaps structural phrases, depending on the specific relation), it suggests that there are natural patterns of spoken phrase rhythms or phrase durations that are best suited for decoding spoken language, driven by the necessity to match a cortical function. Deploying the experimental analysis approach, we describe here to real speech can elucidate the temporal aspects of spoken language comprehension.

\section{Infra- $\delta$ chunking rate}

As discussed earlier we define the relevant $\delta$ range to be between 0.5 and $2 \mathrm{~Hz}$, and chose the $1.8-\mathrm{Hz}$ condition to represent the case where the input chunking rate is inside $\delta$, and the $2.6-\mathrm{Hz}$ condition to represent the outside $\delta$ case. The main research question of our study was whether elicited $\delta$ cortical oscillations correlate with behavior. In particular, does performance deteriorate if the chunk rate is outside the $\delta$ range? We addressed this question by looking at an above- $\delta$ chunking rate $(2.6 \mathrm{~Hz})$, but we did not look at infra- $\delta$ rates (e.g., $0.3 \mathrm{~Hz}$ ). The reason to skip the effects of infra- $\delta$ rates stemmed from the fact that the decay time of sensory memory, $\sim 2 \mathrm{~s}$ long (Cowan, 1984), roughly coincides with the lower bound of the $\delta$-cycle duration. Consequently, the dominant factor at the origin of a possible deterioration in performance may very well be an internal time constraint on processing spoken material (because of echoic memory span) rather than prosodic segmentation.

In conclusion, oscillation-based models of speech perception (Ghitza, 2011; Giraud and Poeppel, 2012; Gross et al., 2013; Martin and Doumas, 2017; Haegens and Zion Golumbic, 2018; Rimmele et al., 2018b; Lakatos et al., 2019) postulate a cortical computational principle by which decoding is performed within a time-varying window structure, synchronized with the input on multiple time scales. The windows are generated by a segmentation process, implemented by a cascade of oscillators. At the prelexical level, the segmentation process is realized by a flexible $\theta$ oscillator locked to the input syllabic rhythm, where the $\theta$ cycles constitute the syllabic windows. Doelling et al. (2014) provided MEG evidence for the role of $\theta$, showing that intelligibility is correlated with the existence of acoustic-driven $\theta$ neuronal oscillations.

Our major finding, that phrase-size chunking of digit strings is correlated with acoustic-driven $\delta$ oscillations, suggests that the role played by neuronal $\theta$-band oscillations in syllabic segmentation can be generalized to the phrasal time scale. The segmentation process is realized by a flexible $\delta$ oscillator locked to the input phrase-size chunk rhythm, where the $\delta$ cycles constitute the phrasesize chunk windows.

Future research is required to investigate whether our findings can be generalized to continuous speech (i.e., beyond digit strings). That is, whether the intonational phrase patterns of language could be constrained by cortical $\delta$ oscillations. Adopting the view that the strategy of composing syllables and words into phrasal units is the result of an evolutionary trajectory to match a cortical function (Patel and Iversen, 2014; Bosman and Aboitiz, 2015), we hypothesize that the phrases of language are constrained by $\delta$ oscillations, and the rules of chunking in speech production may be the product of common cortical mechanisms on both motor and sensory sides, with $\delta$ at the core.

\section{References}

Ahissar E, Ahissar M (2005) Processing of the temporal envelope of speech. In: The auditory cortex: a synthesis of human and animal research, pp 295-314. Mahwah: Erlbaum.

Assaneo MF, Rimmele JM, Orpella J, Ripollés P, de Diego-Balaguer $R$, Poeppel D (2019) The lateralization of speech-brain coupling is differentially modulated by intrinsic auditory and top-down mechanisms. Front Integr Neurosci 13:28.

Assaneo MF, Rimmele JM, Sanz Perl Y, Poeppel D (2020) Speaking rhythmically can shape hearing. Nat Hum Behav 5:71-82.

Aubanel V, Davis C, Kim J (2016) Exploring the role of brain oscillations in speech perception in noise: intelligibility of isochronously retimed speech. Front Hum Neurosci 10:430.

Auer P, Couper-Kuhlen E, Müller F (1999) Language in time: the rhythm and tempo of spoken interaction. New York: Oxford University Press.

Binder JR, Desai RH, Graves WW, Conant LL (2009) Where is the semantic system? A critical review and meta-analysis of 120 functional neuroimaging studies. Cereb Cortex 19:2767-2796.

Bonhage CE, Meyer L, Gruber T, Friederici AD, Mueller JL (2017) Oscillatory EEG dynamics underlying automatic chunking during sentence processing. Neuroimage 152:647-657.

Bosman CA, Aboitiz F (2015) Functional constraints in the evolution of brain circuits. Front Neurosci 9:303.

Boucher VJ, Gilbert AC, Jemel B (2019) The role of low-frequency neural oscillations in speech processing: revisiting delta entrainment. J Cogn Neurosci 31:1205-1215.

Brainard DH (1997) The psychophysics toolbox. Spat Vis 10:433436.

Breen M (2018) Effects of metric hierarchy and rhyme predictability on word duration in The Cat in the Hat. Cognition 174:71-81.

Breska A, Deouell LY (2017) Neural mechanisms of rhythm-based temporal prediction: delta phase-locking reflects temporal predictability but not rhythmic entrainment. PLoS Biol 15:e2001665.

Bröhl F, Kayser C (2020) Delta/theta band EEG differentially tracks low and high frequency speech envelopes. bioRxiv 2020.07.26.221838.

Brungart D, van Wassenhove V, Brandewie E, Romigh GD (2007) The effects of temporal acceleration and deceleration on AV speech perception. International Conference on Auditory-Visual Speech Processing (AVSP), Hilvarenbeek, The Netherlands. 
Buiatti M, Peña M, Dehaene-Lambertz G (2009) Investigating the neural correlates of continuous speech computation with frequency-tagged neuroelectric responses. Neuroimage 44:509519.

Capilla A, Pazo-Alvarez P, Darriba A, Campo P, Gross J (2011) Steady-state visual evoked potentials can be explained by temporal superposition of transient event-related responses. PLoS One 6:e14543.

Clifton CJ, Carlson K, Frazier L (2006) Tracking the what and why of speakers' choices: prosodic boundaries and the length of constituents. Psychon Bull Rev 13:854-861.

Cowan N (1984) On short and long auditory stores. Psychol Bull 96:341-370.

Daume J, Wang P, Maye A, Zhang D, Engel AK (2021) Non-rhythmic temporal prediction involves phase resets of low-frequency delta oscillations. Neuroimage 224:117376.

Deniz ND, Fodor JD (2019) Timing of syntactic and rhythmic effects on ambiguity resolution in Turkish: a phoneme restoration study. Lang Speech 63:832-855.

Ding N, Simon JZ (2009) Neural representations of complex temporal modulations in the human auditory cortex. J Neurophysiol 102:2731-2743.

Ding N, Melloni L, Zhang H, Tian X, Poeppel D (2016) Cortical tracking of hierarchical linguistic structures in connected speech. Nat Neurosci 19:158-164.

Doelling KB, Arnal LH, Ghitza O, Poeppel D (2014) Acoustic landmarks drive delta-theta oscillations to enable speech comprehension by facilitating perceptual parsing. Neuroimage 85:761-768.

Doelling KB, Assaneo MF, Bevilacqua D, Pesaran B, Poeppel D (2019) An oscillator model better predicts cortical entrainment to music. Proc Natl Acad Sci USA 116:10113.

Donhauser PW, Baillet S (2020) Two distinct neural timescales for predictive speech processing. Neuron 105:385-393.e9.

Flinker A, Doyle WK, Mehta AD, Devinsky O, Poeppel D (2019) Spectrotemporal modulation provides a unifying framework for auditory cortical asymmetries. Nat Hum Behav 3:393-405.

Miller G (1962) Decision units in the perception of speech. IEEE Trans Inform Theory 8:81-83.

Gee JP, Grosjean F (1983) Performance structures: a psycholinguistic and linguistic appraisal. Cognit Psychol 15:411-458.

Ghitza O (2011) Linking speech perception and neurophysiology: speech decoding guided by cascaded oscillators locked to the input rhythm. Front Psychol 2:1-13.

Ghitza O (2014) Behavioral evidence for the role of cortical theta oscillations in determining auditory channel capacity for speech. Front Psychol 5:652.

Ghitza O (2017) Acoustic-driven delta rhythms as prosodic markers. Lang Cogn Neurosci 32:545-561.

Ghitza O (2020) "Acoustic-driven oscillators as cortical pacemaker": a commentary on Meyer, Sun and Martin (2019). Lang Cogn Neurosci 35:1100-1105.

Ghitza O, Greenberg S (2009) On the possible role of brain rhythms in speech perception: intelligibility of time-compressed speech with periodic and aperiodic insertions of silence. Phonetica 66:113-126.

Giraud AL, Poeppel D (2012) Cortical oscillations and speech processing: emerging computational principles and operations. Nat Neurosci 15:511-517.

Green DM, Swets JA (1966) Signal detection theory and psychophysics. New York: Wiley.

Gross J, Hoogenboom N, Thut G, Schyns P, Panzeri S, Belin P, Garrod S (2013) Speech rhythms and multiplexed oscillatory sensory coding in the human brain. PLoS Biol 11:e1001752.

Haegens S, Zion Golumbic E (2018) Rhythmic facilitation of sensory processing: a critical review. Neurosci Biobehav Rev 86:150-165.

Henin S, Turk-Browne NB, Friedman D, Liu A, Dugan P, Flinker A, Doyle W, Devinsky O, Melloni L (2021) Learning hierarchical sequence representations across human cortex and hippocampus. Sci Adv 7:eabc4530.
Hickok G, Poeppel D (2007) The cortical organization of speech processing. Nat Rev Neurosci 8:393-402.

Hilton CB, Goldwater M (2020) Linguistic syncopation: Meter-syntax alignment affects sentence comprehension and sensorimotor synchronization. PsyArXiv. doi: 10.31234/osf.io/hcngm.

Hovsepyan S, Olasagasti I, Giraud AL (2020) Combining predictive coding and neural oscillations enables online syllable recognition in natural speech. Nat Commun 11:3117.

Inbar M, Grossman E, Landau AN (2020) Sequences of Intonation Units form a $\sim 1 \mathrm{~Hz}$ rhythm. Sci Rep 10:15846.

JASP Team (2020) JASP (version 0.12)[Computer software].

Jepsen ML, Ewert SD, Dau T (2008) A computational model of human auditory signal processing and perception. J Acoust Soc Am 124:422-438.

Keitel A, Gross J, Kayser C (2018) Perceptually relevant speech tracking in auditory and motor cortex reflects distinct linguistic features. PLoS Biol 16:e2004473.

Kösem A, Bosker HR, Takashima A, Meyer A, Jensen O, Hagoort P (2018) Neural entrainment determines the words we hear. Curr Biol 28:2867-2875.e3.

Lakatos P, Shah AS, Knuth KH, Ulbert I, Karmos G, Schroeder CE (2005) An oscillatory hierarchy controlling neuronal excitability and stimulus processing in the auditory cortex. I Neurophysiol 94:1904-1911.

Lakatos P, Gross J, Thut G (2019) A new unifying account of the roles of neuronal entrainment. Curr Biol 29:R890-R905.

Makeig S, Bell AJ, Jung TP, Sejnowski TJ (1996) Independent component analysis of electroencephalographic data. Adv Neural Inf Process Syst 8:145-151.

Martin AE, Doumas LAA (2017) A mechanism for the cortical computation of hierarchical linguistic structure. PLoS Biol 15:e2000663.

Martin P (2015) The structure of spoken language: intonation in romance. Cambridge: Cambridge University Press.

Meyer L, Henry M, Gaston P, Schmuck N, Friederici A (2017) Linguistic bias modulates interpretation of speech via neural deltaband oscillations. Cereb Cortex 27:4293-4302.

Morillon B, Arnal LH, Schroeder CE, Keitel A (2019) Prominence of delta oscillatory rhythms in the motor cortex and their relevance for auditory and speech perception. Neurosci Biobehav Rev 107:136-142.

Nolte G (2003) The magnetic lead field theorem in the quasi-static approximation and its use for magnetoencephalography forward calculation in realistic volume conductors. Phys Med Biol 48:3637.

Nourski KV, Reale RA, Oya $\mathrm{H}$, Kawasaki H, Kovach CK, Chen $\mathrm{H}$, Howard MA, Brugge JF (2009) Temporal envelope of time-compressed speech represented in the human auditory cortex. J Neurosci 29:15564.

Oganian Y, Chang EF (2019) A speech envelope landmark for syllable encoding in human superior temporal gyrus. Sci Adv 5: eaay6279.

Oldfield RC (1971) The assessment and analysis of handedness: the Edinburgh inventory. Neuropsychologia 9:97-113.

Oostenveld R, Fries P, Maris E, Schoffelen JM (2011) FieldTrip: open source software for advanced analysis of MEG, EEG, and invasive electrophysiological data. Intell Neurosci 2011:156869.

Park H, Ince RAA, Schyns PG, Thut G, Gross J (2015) Frontal topdown signals increase coupling of auditory low-frequency oscillations to continuous speech in human listeners. Curr Biol 25:16491653.

Patel AD, Iversen JR (2014) The evolutionary neuroscience of musical beat perception: the action simulation for auditory prediction (ASAP) hypothesis. Front Syst Neurosci 8:57.

Peelle JE, Davis MH (2012) Neural oscillations carry speech rhythm through to comprehension. Front Psychol 3:320.

Pittman-Polletta BR, Wang Y, Stanley DA, Schroeder CE, Whittington MA, Kopell NJ (2021) Differential contributions of synaptic and intrinsic inhibitory currents to speech segmentation via flexible phase-locking in neural oscillators. PLOS Comput Biol 17:e1008783. 
Poeppel D (2003) The analysis of speech in different temporal integration windows: cerebral lateralization as 'asymmetric sampling in time'. Nat Speech Percept 41:245-255.

Rimmele JM, Gross J, Molholm S, Keitel A (2018a) Editorial: brain oscillations in human communication. Front Hum Neurosci 12:39.

Rimmele JM, Morillon B, Poeppel D, Arnal LH (2018b) Proactive sensing of periodic and aperiodic auditory patterns. Trends Cogn Sci 22:870-882.

Rimmele JM, Poeppel D, Ghitza O (2020) Acoustically driven cortical delta oscillations underpin perceptual chunking. bioRxiv 2020.05.16.099432.

Schönbrodt FD, Wagenmakers EJ (2018) Bayes factor design analysis: planning for compelling evidence. Psychon Bull Rev 25:128-142.

Shattuck-Hufnagel S, Turk AE (1996) A prosody tutorial for investigators of auditory sentence processing. J Psycholinguist Res 25:193-247.

Stehwien S, Meyer L (2021) Rhythm Comes, Rhythm Goes: Shortterm Periodicity of Prosodic Phrasing. PsyArXiv. doi: 10.31234/ osf.io/c9sgb.
Sternberg S (1966) High-speed scanning in human memory. Science 153:652-654.

Stolk A, Todorovic A, Schoffelen J-M, Oostenveld R (2013) Online and offline tools for head movement compensation in MEG. Neuroimage 68:39-48.

Tzourio-Mazoyer N, Landeau B, Papathanassiou D, Crivello F, Etard O, Delcroix N, Mazoyer B, Joliot M (2002) Automated anatomical labeling of activations in SPM using a macroscopic anatomical parcellation of the MNI MRI single-subject brain. Neuroimage 15:273-289.

Van Veen BD, van Drongelen W, Yuchtman M, Suzuki A (1997) Localization of brain electrical activity via linearly constrained minimum variance spatial filtering. IEEE Trans Biomed Eng 44:867880.

Wang Y, Ding N, Ahmar N, Xiang J, Poeppel D, Simon JZ (2011) Sensitivity to temporal modulation rate and spectral bandwidth in the human auditory system: MEG evidence. J Neurophysiol 107:2033-2041. 